\title{
Large-area travelling-wave dielectrophoresis particle separator
}

\author{
H Morgan, N G Green, M P Hughes, W Monaghan and T C Tan
}

Bioelectronics Research Centre, Department of Electronics and Electrical Engineering, University of Glasgow, Glasgow G12 8LT, UK

Received 13 August 1996, accepted for publication 25 February 1997

\begin{abstract}
Multilayering microelectrode techniques have been developed to construct large-area travelling-wave dielectrophoresis (TWD) separators. Novel electrode designs have enabled arrays with up to 25000 electrodes to be constructed. The separation capacity of a 1000 electrode $(20 \mathrm{~mm})$ array has been demonstrated by separating components of whole blood. The development of sophisticated bio-particle separators capable of separating cells, viruses and proteins is discussed.
\end{abstract}

\section{Introduction}

The principle of using sequentially-addressed arrays of parallel microelectrodes to electrostatically propel microparticles, originally described by Masuda et al $[1,2]$, has now been developed into a technique for characterizing and separating cells and micro-organisms [3-6]. Travelling electric fields can also be used as electrohydrodynamic pumps capable of moving liquids [7]. In initial experiments low frequency travelling electric fields were observed to induce motion in blood cells via largely electrophoretic interactions between the cell and the electrodes [1]. Later work, using fields of much higher frequency, showed that an asynchronous motive force could be induced in latex beads [3]. This force arises from a displacement between the induced dipole moment of the particle and the applied travelling electric field, in a linear equivalent to classical electrorotation [8]. In a study by Huang et al in 1993 [6] this linear force was termed travelling-wave dielectrophoresis (TWD).

Theoretical studies $[5,6,9,10]$ have shown that the translational force experienced by a particle undergoing TWD depends on a number of factors: the particle volume, the particle and medium polarizabilities, the electric field strength and the wavelength of the travelling field. For a given particle size and applied field strength, the absolute force on a particle is determined by the difference between the imaginary parts of the polarizabilities of the particle and the suspending medium. Therefore, it is possible to separate a dielectrically heterogeneous population of particles (equally sized) into homogeneous subpopulations as has been reported previously [6].

Practical TWD particle separators for biotechnological applications have yet to be developed owing to the small scale of the electrode arrays used (typically 70 electrodes in length). This paper describes the design and construction of a large-scale TWD array, consisting of 1000 individual
$10 \mu \mathrm{m}$ wide electrodes, fabricated using multilayering techniques. This electrode array is used to demonstrate the potential of TWD as a technique for rapid and highresolution bio-particle separation.

\section{Theory of operation}

An electric field $\boldsymbol{E}$, will induce a dipole moment $\boldsymbol{m}(t)$ in a polarizable particle. At certain frequencies the dipole moment is in opposition to the applied field, causing negative DEP (repulsion from the electrodes). In a travelling electric field particles experience a linear force. In the negative DEP frequency regime particles are levitated and experience unhindered linear motion.

From the work of Hughes et al [5], Wang et al [9] and Washizu and Jones [10] the dipole moment $\boldsymbol{m}(t)$ can be defined as follows:

$$
\boldsymbol{m}(t)=m_{x}(t) \boldsymbol{a}_{x}+m_{y}(t) \boldsymbol{a}_{y}+m_{z}(t) \boldsymbol{a}_{z}
$$

where $a_{x}, a_{y}, a_{z}$ are the unit vectors in the $x, y$ and $z$ directions, respectively, and $m_{x}(t), m_{y}(t)$ and $m_{z}(t)$ are the magnitudes of the induced dipole moment in each direction. It can be shown [9] that the TWD force exerted on a particle in an electric field, at sufficient distance to minimize higherorder spatial frequency harmonics [5], can be described by

$$
\boldsymbol{F}(t)=\frac{-4 \pi^{2} \varepsilon_{\mathrm{m}} r^{3} \operatorname{Im}\left[f_{C M}\right] \boldsymbol{E}^{2}}{\lambda}
$$

where $\lambda$ is the wavelength of the travelling field and $\operatorname{Im}\left[f_{C M}\right]$ is the imaginary part of the Clausius-Mosotti factor. The real and imaginary parts of $f_{C M}$ give the in-phase and out-of-phase components of the dipole $\boldsymbol{m}(t)$, which determine the conventional dielectrophoretic behaviour and the TWD behaviour, respectively [9]. For a spherical particle, $f_{C M}$ is

$$
f_{C M}=\frac{\varepsilon_{p}^{*}-\varepsilon_{m}^{*}}{\varepsilon_{p}^{*}+2 \varepsilon_{m}^{*}}
$$




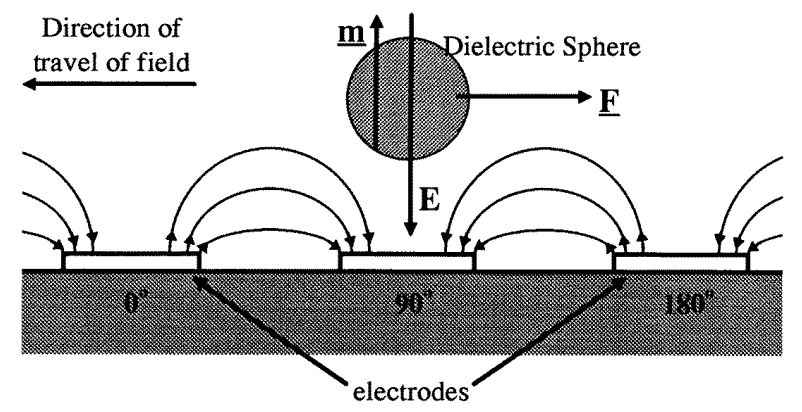

Figure 1. A diagram showing the induced dipole moment $\boldsymbol{m}$ on a particle in a travelling electric field of magnitude $\boldsymbol{E}$. The particle is levitated above the electrodes by negative DEP. A translational force $\boldsymbol{F}$ is induced on the particle due to the phase lag between the field and the dipole.

where $\varepsilon_{p}^{*}$ and $\varepsilon_{m}^{*}$ are the complex permittivities of the particle and suspending medium respectively, given by $\varepsilon^{*}=\varepsilon-j(\sigma / \omega)$, where $\varepsilon$ is the permittivity, $\sigma$ is the conductivity and $j=\sqrt{-1}$. For non-spherical particles, $f_{C M}$ is a function of geometry [11] and as no solution for biconcave discs such as erythrocytes exists, approximations in spherical terms must be made.

As shown in [6], the linear dielectrophoretic force is balanced by viscous drag (given by Stoke's equation). So, considering TWD in a medium of viscosity $\eta$, the velocity $u$ of a particle travelling along the array is given by

$$
u=-\frac{2 \pi \varepsilon_{m} r^{2}}{3 \lambda \eta} \operatorname{Im}\left[f_{C M}\right] \boldsymbol{E}^{2}
$$

The velocity is proportional to the particle radius squared, the electric field strength squared, the travelling field wavelength, the viscosity of the medium and the imaginary part of the Clausius-Mosotti factor.

\section{Electrode design and fabrication}

Figure 2(a) is a schematic diagram of a 10 electrode single-layer TWD array, with each electrode individually connected to a bonding pad. In this figure, each pad and electrode pair is connected sequentially to one phase of a four-phase AC signal but, in general, the signal can be $n$-phase with $n>2$. This type of TWD array can be manufactured easily using conventional electrode microfabrication techniques. However, the fabrication of arrays with greater than (about) 100 electrodes becomes impractical because of the need for the independent connection of each electrode to the signal source. As a consequence, to make TWD arrays suitable for largescale biotechnological applications, new microfabrication techniques based on multilayering technology have been developed.

Figure 2(b) is a schematic outline of an electrode constructed using multilayer methods similar to the production of double-sided printed circuit boards. The four-phase signal is carried across the device by busbars which are separated from the electrodes by a thin layer of insulator. Every fourth electrode is connected through the insulator to one of the busbars. Only four bonding pads are required and the number of electrodes in the array is limited by the physical size of the device. For example, a $100 \mathrm{~mm}$ long array would have $500010 \mu \mathrm{m}$-wide electrodes.

Figure 3 shows the steps in the fabrication of the array. (a) Four busbars (titanium-palladium-gold) are deposited onto a glass substrate using photolithography, metal evaporation and lift-off. (b) Silicon nitride (100 nm thick) is deposited over the whole substrate and then (c) $10 \mu \mathrm{m}$ diameter holes are etched using reactive ion etching. These holes were used to form connections between the busbars and the electrodes by one of two methods. Method 1 involves plating gold through the holes (electrolytically from a gold cyanide solution) to form small mushroomshaped studs (d). Finally the electrodes are fabricated over these studs using photolithography (e). Method 2 was to form the connection during the fabrication of the electrodes using evaporation of metal through the holes. Method 2 was used most frequently owing to its simplicity. If required silicon nitride could be deposited over the entire electrode (f) to protect the electrodes from corrosion by electrolysis at low frequencies.

The array was designed with built-in redundancy, so that open circuits, either in the busbars or the electrodes, did not prevent the device from functioning. The busbars were connected via the bonding pads to the signal with the phases ordered $0^{\circ}-180^{\circ}-90^{\circ}-270^{\circ}$ to avoid the generation of travelling fields between adjacent busbars.

A photograph of a fabricated large-scale TWD electrode array is shown in figure 4 . The array is $2 \mathrm{~cm}$ long and consists of 1000 electrodes, each $10 \mu \mathrm{m}$ wide with a $10 \mu \mathrm{m}$ gap. This particular array was constructed with parallel fingers (as shown in figure 2(b)) and also with a $40 \mu \mathrm{m}$ wide channel between the opposing sets of electrodes as in [6]. Arrays consisting of 25 sets of 1000-electrode TWD separators lying parallel to each other, covering $2.5 \times 2.5 \mathrm{~cm}^{2}$ of substrate were also fabricated, having 25000 electrodes connected to four bonding pads.

The electrodes were addressed with a four-phase AC voltage derived from a direct digital synthesis generator (Qualcomm Q2334 Dual DDS) constructed in-house and operating under computer control. Frequencies could be varied between $100 \mathrm{~Hz}$ and $20 \mathrm{MHz}$ with voltages up to $10 \mathrm{~V}$ peak-to-peak.

\section{Results and discussion}

The capability of the array for bio-particle separation was demonstrated by separating the components of whole rabbit blood using a 1000 electrode array. $10 \mathrm{ml}$ of fresh rabbit blood was collected into heparinized tubes by venous puncture. The blood was centrifuged $(250 \times \mathrm{g})$ and washed twice in $100 \mathrm{mM}$ phosphate buffered saline (PBS) before final re-suspension in $100 \mathrm{mM}$ PBS to a cell density of $5 \times 10^{9}$ cells $\mathrm{ml}^{-1}$. Mononuclear cells were separated from the erythrocytes by centrifugation in Histopaque1077 (Sigma Diagnostics, Poole, Dorset, UK) following the protocol provided by the manufacturers. Erythrocytes and leukocytes were re-suspended in a ratio of 10:1, in a buffer consisting of $10 \%$ PBS, 90\% $280 \mathrm{mM}$ Mannitol to 


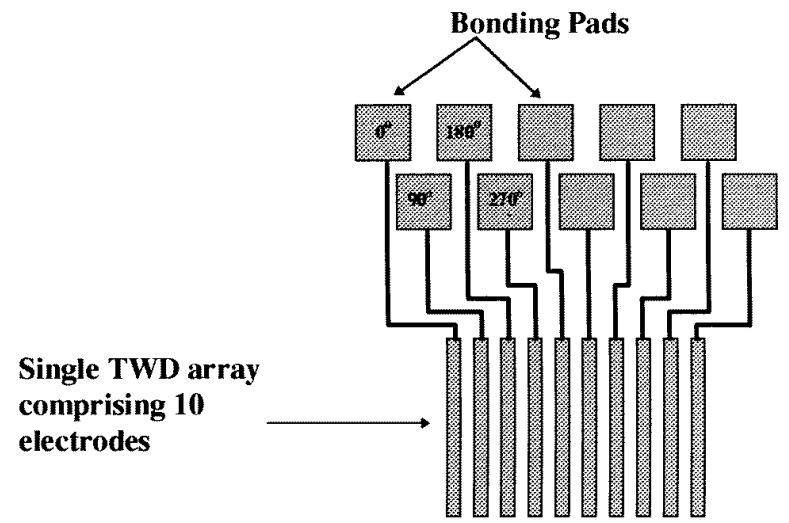

(a)

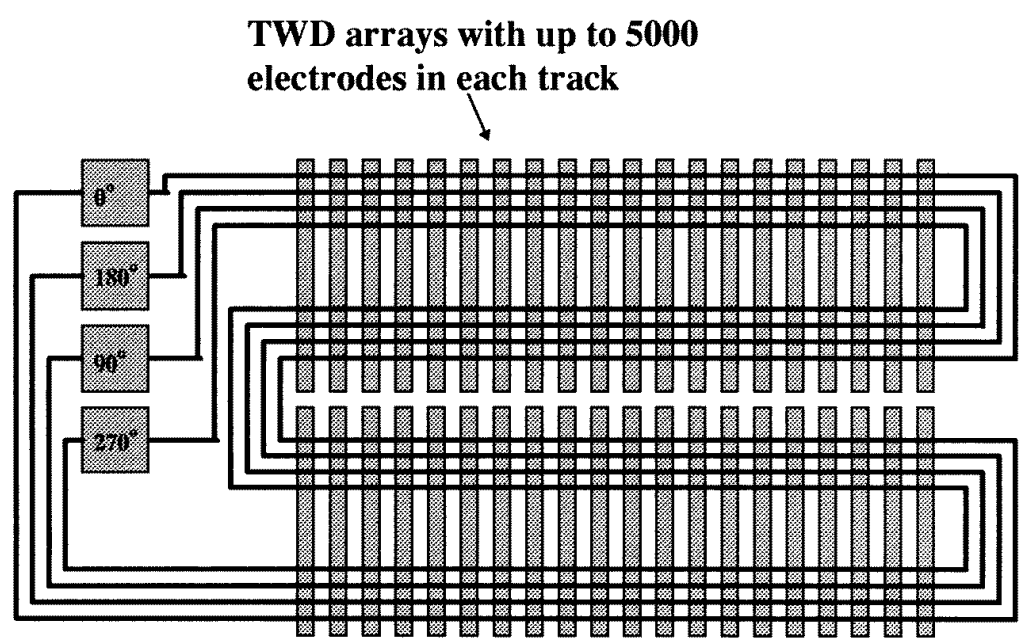

(b)

Figure 2. A schematic drawing of two designs of travelling-wave dielectrophoresis arrays for operation with a four-phase signal. (a) shows a 10 electrode array constructed using conventional single layer lithography with each electrode separately connected to one of four phases. In contrast, (b) shows a design for an array constructed using multilayering techniques. The number of electrodes is limited only by the size of the substrate.

a final cell density of $5 \times 10^{7}$ cells $\mathrm{ml}^{-1}$. The conductivity of this solution was $150 \mathrm{mS} \mathrm{m} \mathrm{m}^{-1}$. A $10 \mu \mathrm{l}$ aliquot of the solution was micropipetted onto the electrode array and the surface covered with a microscope cover slip. Regions of the electrode array were imaged using a Nikon Microphot microscope and images of moving cells were recorded on video.

A four-phase signal of $10 \mathrm{~V}$ peak-to-peak at a frequency of $500 \mathrm{kHz}$ was used to linearly translate blood along the TWD electrode array. At this frequency and voltage, there was a distinct difference between the velocities of different types of cells. Figure 5 shows a section of the TWD array with blood cells moving across the field of view from left to right, the four images taken at $1 \mathrm{~s}$ intervals. During this time, the erythrocyte had moved $90 \mu \mathrm{m}$ and the leukocyte had moved approximately $60 \mu \mathrm{m}$. The electrodes are out of focus in this figure owing to the fact that the cells are levitated by approximately $7 \mu \mathrm{m}$ by negative dielectrophoretic forces [6]. Observation of the movement of several cells across a number of $100 \mu \mathrm{m}$ wide frames, gave mean velocities of $32 \mu \mathrm{m} \mathrm{s}^{-1}$ for the erythrocytes and $20 \mu \mathrm{m} \mathrm{s}^{-1}$ for the leukocytes. The particles were observed to move in a direction opposite to the travelling electric field as predicted by equation (2). A simple analysis of the separation efficiency shows that, assuming both cell types begin travelling at the same time and from the same end of the array, there would be a path difference of $7.5 \mathrm{~mm}$ by the time the erythrocytes reach the end of the electrodes.

The efficiency of separation for two given particle types depends on the length of the array, all other factors being equal. For the erythrocytes and leukocytes, the mixtures of cells would have separated into two homogeneous population bands separated spatially by $1 \mathrm{~mm}$ after just $80 \mathrm{~s}$, the erythrocytes having travelled $2.56 \mathrm{~mm}$. For the separation of small numbers of cells, as required for diagnostic purposes, smaller band separations would be 


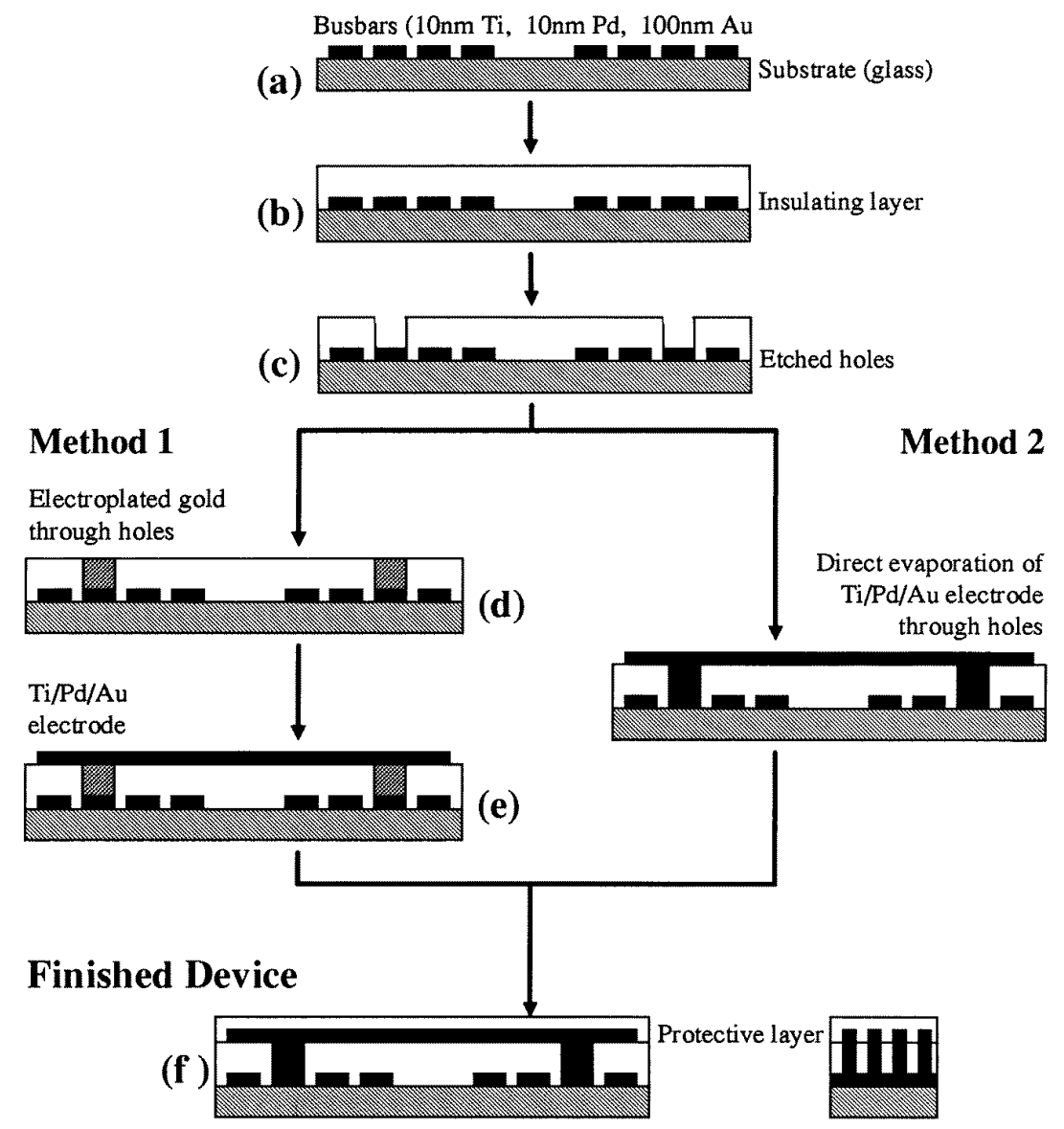

Figure 3. A diagram showing the consecutive steps in the construction of a TWD electrode array using multilayering. (a) Fabrication of busbars on glass substrate is followed by (b) deposition of silicon nitride insulator. (c) Holes are produced using reactive ion etching through a masking pattern and the electrodes and the connections to the busbars are fabricated either by plating of gold through the holes (method 1) or direct evaporation through the holes followed by lift-off (method 2).
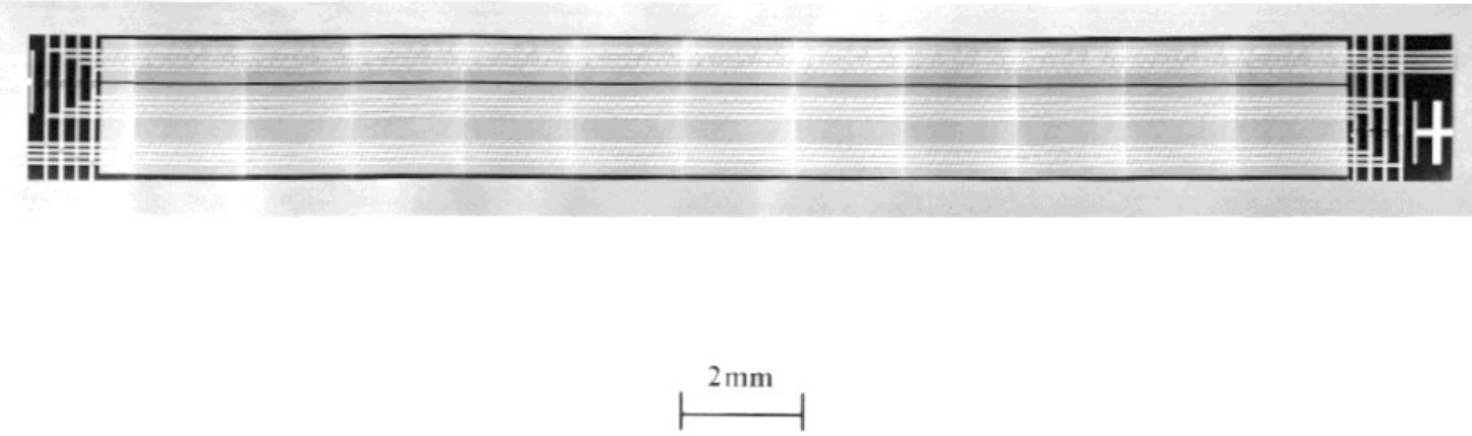

Figure 4. Photograph of a 1000 electrode TWD array. The electrodes are $10 \mu \mathrm{m}$ wide separated by $10 \mu \mathrm{m}$ gaps and the total length of the array was $20 \mathrm{~mm}$. This array was fabricated to include a $40 \mu \mathrm{m}$ gap between opposing sets of electrodes [6]. The photograph is a composite of several images taken through a microscope.

acceptable. Therefore, for large TWD arrays extending over the length of a glass microscope slide $(50 \mathrm{~mm})$ it would be possible to fraction cells with extremely small differences in dielectric properties $\left(\operatorname{Im}\left[f_{C M}\right]\right)$. For an array $5 \mathrm{~cm}$ long, an equivalent change in $\operatorname{Im}\left[f_{C M}\right]$ of as little as $0.2 \%$ would translate into a band separation of $100 \mu \mathrm{m}$.

For any two dielectrically equivalent particles, from equation (4), the travelling velocity is directly proportional to the particle radius squared and the square of the electric field. It follows that, in order to separate submicrometresized particles such as viruses or macromolecules, the electric field must be increased to compensate for the reduction in size. Generating high voltages at frequencies up to $20 \mathrm{MHz}$ is impractical and the only alternative therefore is to fabricate electrodes of much smaller dimensions using nanolithographic techniques. 

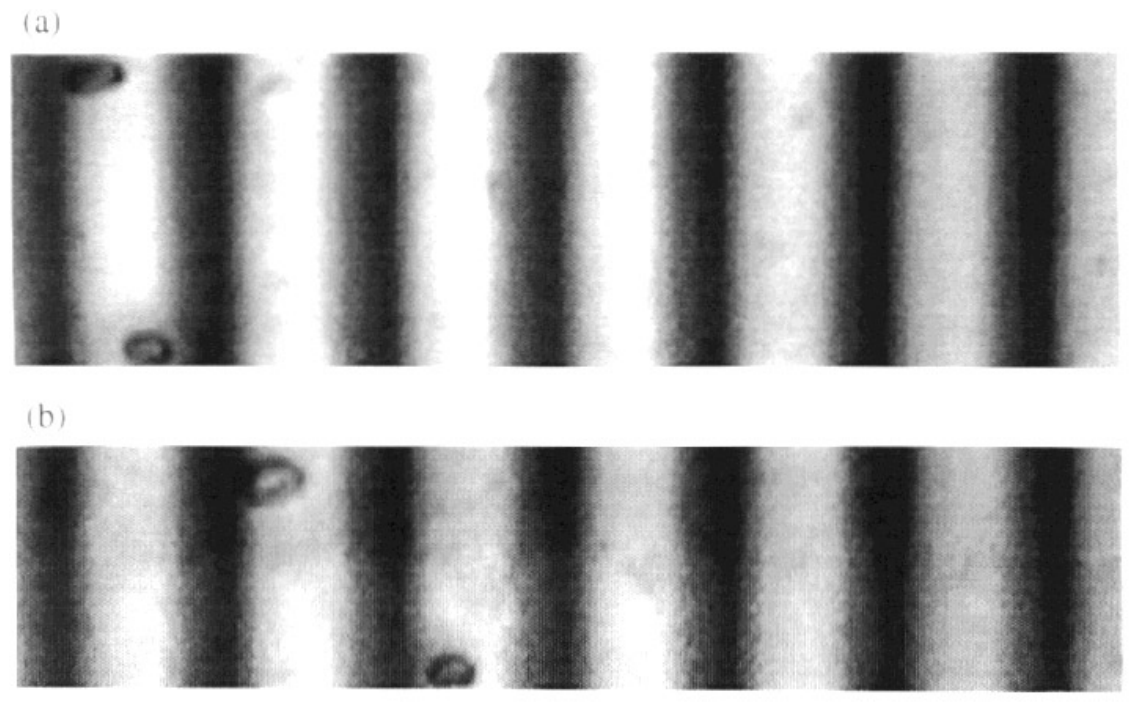

(c)
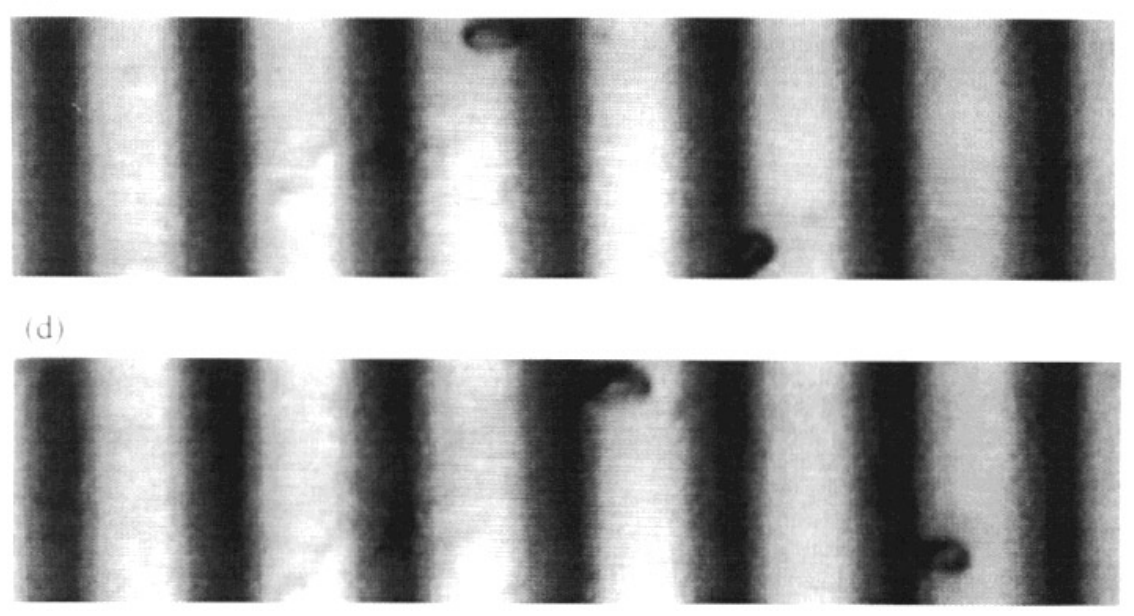

Figure 5. A sequence of four images taken at $1 \mathrm{~s}$ intervals (a, b, c, d) showing an erythrocyte overtaking a leukocyte. The frame width is approximately $140 \mu \mathrm{m}$. The cells are levitated above the plane of the electrodes by approximately $7 \mu \mathrm{m}$.

Recently, Washizu [12] reported observations of the molecular dielectrophoresis (as opposed to TWD) of biopolymers and was able to successfully demonstrate the separation of both proteins and DNA. In this case, the effect was governed by $\operatorname{Re}\left[f_{C M}\right]$ (the permittivity of the particle) and equation (4) shows that TWD is governed by $\operatorname{Im}\left[f_{C M}\right]$ (the conductivity of the particle). Therefore, by combining dielectrophoretic separators and TWD array separators, it should be possible to separate a whole range of bio-particles, ranging from cells and bacteria to viruses and chromosomes, and even proteins and DNA.

\section{Conclusions}

Travelling-wave dielectrophoresis (TWD) is a powerful means of manipulating micrometre and submicrometresized particles suspended in fluids of low conductivity. The linear force exerted on a given particle is dependent not only on its size but also on $\operatorname{Im}\left[f_{C M}\right]$ which, in turn, depends on the conductivity of the particle. By careful selection of the suspending medium, it is theoretically possible to separate particles with very small differences in dielectric properties into homogeneous populations.

\section{Acknowledgments}

This work was supported by the Biotechnology and Biological Sciences Research Council (grant No: 17/T05315). The authors wish to thank Professor CDW Wilkinson and Dr Simon Hicks for silicon nitride fabrication and Ms Mary Robertson for preparation of blood.

\section{References}

[1] Masuda S, Washizu M and Iwadare M 1987 Separation of small particles suspended in liquid by non-uniform travelling field IEEE Trans. Ind. Appl. 23 474-80

[2] Masuda S, Washizu M and Kawabata I 1988 Movement of blood cells in liquid by non-uniform travelling field IEEE Trans. Ind. Appl. 24 217-22

[3] Fuhr G, Hagedorn R, Muller T, Benecke W, Wagner B and Gimsa J 1991 Asynchronous travelling-wave induced linear motion of living cells Studia Biophys. 140 79-102 
[4] Hagedorn R, Fuhr G, Muller T and Gimsa J 1992 Travelling wave dielectrophoresis of microparticles Electrophoresis 13 49-54

[5] Hughes M P, Pethig R and Wang X-B 1996 Dielectrophoretic forces on particles in travelling electric fields J. Phys. D: Appl. Phys. 29 474-82

[6] Huang Y, Wang X-B, Tame J and Pethig R 1993 Electrokinetic behaviour of colloidal particles in travelling electric fields: studies using yeast cells $J$. Phys. D: Appl. Phys. 26 312-22

[7] Fuhr G, Hagedorn R, Muller T, Benecke W and Wagner B 1992 Microfabricated electrohydrodynamic (EHD) pumps for liquids of higher conductivity $J$. Microelectromech. Syst. 1 141-6

[8] Arnold W M and Zimmerman U 1982

Rotating-field-induced rotation and measurement of the membrane capacitance of single mesophyll-cells of avena-sativa Z. Naturf. C 37 908-15
[9] Wang X-B, Huang Y, Becker F F and Gascoyne P R C 1994 A unified theory of dielectrophoresis and travelling-wave dielectrophoresis J. Phys. D: Appl. Phys. 27 1571-4

[10] Washizu M and Jones T B 1996 Generalised multipolar dielectrophoretic force and electrorotational torque calculation J. Electrostat. 38 199-211

[11] Kakutani T, Shibatani S and Sugai M 1993 Electrorotation of non-spherical cells: theory for ellipsoidal cells with an arbitrary number of shells Bioelectrochem. Bioenerg. 31 131-45

[12] Washizu M, Suzuki S, Kurosawa O, Nishizaka T and Shinohara T 1994 Molecular dielectrophoresis of biopolymers IEEE Trans. Ind. Appl. 30 835-43 